\title{
On the Reactions $A+A+\ldots+A \rightarrow 0$ at a One-Dimensional Periodic Lattice of Catalytic Centers: Exact Solution
}

\author{
A.A.Naidenov ${ }^{1}$ and S.K.Nechaev ${ }^{2,1}$ \\ ${ }^{1}$ L.D.Landau Institute for Theoretical Physics RAN, \\ Kosygin str. 2, 117334 Moscow, Russia \\ ${ }^{2}$ Laboratoire de Physique Théorique et Modéles Statistiques, \\ Université Paris Sud, 91405 Orsay Cedex, France
}

(Published in JETP Letters, 76 (2002), 61-65)

\begin{abstract}
The kinetics of the diffusion-controlled chemical reactions $A+A+\ldots+A \rightarrow 0$ that occur at catalytic centers periodically arranged along a straight line is considered. Modes of the behavior of reaction probability $W(t)$ were studied at different times and different densities of the catalyst. Within the Smoluchowski approximation, it was rigorously proved that at large times the function $W(t)$ is independent of the lattice period. This means that, in the given asymptotic mode, the probability of the reaction on a lattice with a catalyst placed in each lattice site is the same as on a lattice with a catalyst placed in sparse sites
\end{abstract}

In this work, we discuss the kinetics of the polymolecular irreversible diffusion-controlled chemical reactions $A+A+\ldots+A \rightarrow 0$ that occur at catalytic centers arranged in a periodic lattice in space. To our knowledge, the problem of the decrease in the amount of the reactant with time in catalytic diffusion-controlled chemical reactions $A+A \rightarrow 0$ was first studied within the random phase approximation in [1].

The problem considered in this article "rests on two whales": on the one hand, it has characteristic features of the dynamics of diffusion-controlled chemical reactions, and, on the other hand, it is typical for the adsorption kinetics of particles diffusing in the medium of stationary "traps" (absorbers). Before going into the description of the model under study, let us briefly formulate the main features of its fundamental problems: diffusion-controlled chemical reactions and kinetics of the adsorption of particles on traps.

For several decades, bimolecular diffusion-controlled reactions $A+A \rightarrow B$ served as the basic model for studying the kinetics of chemical reactions on the microscopic scale. The main ideas of the description of chemical reactions in the medium of diffusing reactants were formulated by Smoluchowski in 1917 [2]. Mean field methods, which were proposed by Smoluchowski and further developed by his numerous disciples (see, e.g., review [3]) are widely used up to now 
and yield reasonable results for problems related to the determination of the rate of chemical reactions and change in the concentration of the reactant with time. However, in the case of strong stirring, i.e., when the diffusion of particles is significant, mean field methods become inappropriate because, along with pair interactions of particles, correlations that occur at triple, quadruple, etc. particle collisions should be taken into account. In works that date back to the 1980s, fluctuation effects in the kinetics of chemical reactions were included [4]. Later the range of problems under study was signifi- cantly expanded by the inclusion of problems of chem- ical kinetics involving macromolecules, so-called transport-controlled chemical reactions [3]. However, the comparison of the results that were obtained within the Smoluchowski approximation with exact solutions for some model systems, e.g., for a one-dimensional bimolecular reaction $A+A \rightarrow 0$ (without catalyst) demonstrates that the mean field method provides cor- rect scaling for the probability of particle decay at large times [5, 6], which differs from the exact solution only by a numerical coefficient.

The problems of determining the evolution of survival or decay probability for particles diffusing in $d$-dimensional space in the presence of stationary randomly arranged absorber traps is the subject of many studies in both the physicochemical and mathematical literature that have appeared over the last two decades or more. The great interest in this topic is obviously caused by its significance in studying such physical chemistry problems as photoconductivity and photo- synthesis, the kinetics of binding biopolymers with ligands, and the adsorption of polymer molecules on surfaces with chemically active regions and on colloidal particles in solutions and gels. Works dating back to the 1970s [7] demonstrated that the probability of the survival of independent diffusing particles in a medium with absorbing traps at large times cannot be described within the Smoluchowski mean field approximation and is controlled by the diffusion time of a particle in a cavity of typical size free from traps. The method that provides correct asymptotic relationships is identical to the optimal fluctuation method by I.M. Lifshitz for estimating the density of states of a disordered system in the vicinity of the band edge (so-called Lifshitz singularities). Thus, works [7, 3, 国 stimulated the interest of mathematicians in the problem of particle decay in a medium of randomly arranged absorbers. Subsequent studies of model systems demonstrated the relation of this problem to percolation, the statistics of polymers in a medium of random obstacles, and some aspects of supersymmetric quantum mechanics [4, 目, Q, [0].

Now, with the general notion as to what type of problem of statistical physics our problem belongs, we can turn to the discussion of the model, which, as noted above, was formulated in [1]. Let us consider a region ("reservoir") in $d$-dimensional space containing an ensemble of identical particles A of finite size involved in Brownian motion and some quantity of stationary catalytic centers (traps). In the course of a random walk, there is the probability of encountering of $m$ particles. If this encounter takes place at a trap, the elementary event of the chemical reaction

$$
\overbrace{A+A+\ldots+A}^{m} \rightarrow 0
$$

occurs and all the particles decay, i.e., are removed from the reservoir; if particles encounter outside the trap, the reaction does not occur. The task consists in calculating the probability $W(t \mid m)$ of the particle decay within the given time interval $t$ depending on the spatial arrangement of traps. 
The probability of particle decay $W(t \mid m=2)$ for a bimolecular chemical reaction was estimated in [1] within the Smoluchowski mean field theory for the periodic and random arrangements of traps, and the effect of catalytic centers was included in [1] within the random phase approximation. In our work, we restrict our consideration to the case of $d=1$ and the periodic lattice of traps. For this model, the distribution function $W(t \mid m)$ can be determined exactly without any additional assumptions. We emphasize that by the exact solution we mean only the possibility of the rigorous inclusion of the effect of the spatially distributed catalyst on the probability of particle decay $W(t \mid m=2)$. Note that, from the standpoint of real physical problem when a finite concentration $\mathrm{c}$ of a reacting particles occurs in the system, we still remain within the Smoluchowski approximation. At the end of the article, we discuss the range of applicability of the given mean field approach. Although one-dimensional, the problem in hand has a particular physicochemical application. Chemical reactions that occur at cathodes in the presence of a catalyst (platinum particles) can be readily described within the model under consideration [11, 12]. This is related to the fact that on the microscopic scale the cathode is a porous structure with one-dimensional channels in which the catalyst is deposited and the diffusion-controlled chemical reaction occurs. A problem of fundamental importance consists in the optimization of the adsorption process: increasing the rate of the chemical reaction and decreasing the concentration of the expensive catalyst. Let $L$ be the distance between the adjacent traps in a straight line. We denote the time dependence of the coordinates of the particles under consideration by $x_{1}(t), x_{2}(t), x_{m}(t)$. The condition that all particles at the instant of time t occur at an arbitrary trap with the coordinate $n L$ is as follows: $x_{1}(t)=x_{2}(t)==x_{m}(t)=n L$. It is easy to realize that the random walk of $\mathrm{m}$ independent particles is equivalent to the effective one-particle random walk in the $\mathrm{m}$-dimensional Euclidean space $\mathcal{E}_{m}=\left(x_{1}, x_{2}, x_{m}\right)$. Thus, the probability $W(t \mid m)$ of the decay of $\mathrm{m}$ identical independent particles randomly wandering along a straight line at their simultaneous encounter at any of the traps is equivalent to the probability of the first occurrence of the random walk in the $\mathcal{E}_{m}$ space at the instance of time $t$ in an arbitrary trap located in the straight line defined by the equation $x_{1}=x_{2}==x_{m}$. In our case, this statement of the problem is a starting point. The recurrence equation for the probability $W_{t}(\mathbf{x}) \equiv W(t \mid m, \mathbf{x})$ to find the random walk over lattice edges in the $m$-dimensional space $\mathcal{E}_{m}$ in the point $\mathbf{x}$ at the instance of time $t$ has the form

$$
W_{t+1}(\mathbf{x})=D \sum_{\mathbf{u}} W_{t}(\mathbf{x}+\mathbf{u}) \eta(\mathbf{x}+\mathbf{u}),
$$

where $D=\frac{1}{2 m}$ is the diffusion coefficient in $m$-dimensional space, summation is performed over the nearest neighbors, and $\eta()$ is the discrete $\delta$-function, which is defined as follows:

$$
\eta(\mathbf{x})= \begin{cases}0, & \text { if } \mathbf{x} \text { coincides with the trap; } \\ 1, & \text { in all the other cases. }\end{cases}
$$

As usual in translation-invariant problems, it is convenient to transform to the momentum Fourier representation:

$$
W_{t}(\mathbf{k})=\frac{1}{N^{m}} \sum_{\mathbf{x}} W_{t}(x) e^{i \mathbf{k x}},
$$

where

$$
W_{t}(\mathbf{x})=\sum_{\left|k_{1}, \ldots, k_{m}\right|<\pi} W_{t}(\mathbf{k}) e^{-i \mathbf{k x}}, \quad k_{j}= \pm \frac{2 \pi s_{j}}{N}
$$


As a result of the Fourier transform, Eq.(1) yields

$$
W_{t+1}(\mathbf{k})=\frac{1}{2 m} \sum_{\mathbf{u}} e^{-i \mathbf{k u}} \sum_{\mathbf{q}} W_{t}(\mathbf{q}) \eta(\mathbf{k}-\mathbf{q}),
$$

where

$$
\eta(\mathbf{k})=\delta(\mathbf{k})-\frac{1}{N^{m}} \sum_{|n L|<N / 2} e^{i k_{1} n L}
$$

In Eq.(6), we assumed that the straight line in which the traps are located coincides with the $\left[0, x_{1}\right)$ axis in the $\mathcal{E}_{m}$ space. Let us use the equation

$$
\sum_{n=-\frac{N}{2 L}}^{\frac{N}{2 L}} e^{i k_{1} n L}=\frac{N}{L} \sum_{|n|<\frac{L}{2}} \delta\left(k_{1}-\frac{2 \pi n}{L}\right)
$$

in which, for the sake of definiteness, we assume $\left[\frac{N}{2 L}\right] \leq=\frac{N}{2 L}$. Using the latter expression, Eq.(5) can be written in the form

$$
W_{t+1}(k)=\frac{1}{m}\left(\cos k_{1}+\ldots+\cos k_{m}\right)\left\{W_{t}(k)-\frac{1}{N^{m-1} L} \sum_{\substack{q_{2}, \ldots, q_{m} \\|n|<L / 2}} W_{t}\left(k_{1}-\frac{2 \pi n}{L}, q_{2}, \ldots, q_{m}\right)\right\} .
$$

where

$$
-\pi \leq\left\{k_{1}, \ldots, k_{m}\right\}<\pi, \quad-\pi \leq\left\{q_{2}, \ldots, q_{m}\right\}<\pi
$$

Equations of this type are conveniently solved by the generating function method:

$$
W(\mathbf{k}, s)=\sum_{t=0}^{\infty} W_{t}(\mathbf{k}) s^{t}, \quad W(t)=\frac{1}{2 \pi i} \int_{C} \frac{W(s) d s}{s^{t+1}} .
$$

Multiplying both parts of expression (7) by $s^{t}$, after simple algebraic transformations we obtain

$$
W(\mathbf{k}, s)=\frac{W_{0}(\mathbf{k})}{1-A(\mathbf{k}, s)}-\frac{A(\mathbf{k}, s)}{1-A(\mathbf{k}, s)} \frac{S\left(\frac{W_{0}(\mathbf{k})}{1-A(\mathbf{k}, s)}\right)}{S\left(\frac{1}{1-A(\mathbf{k}, s)}\right)}
$$

where the following designations are introduced:

$$
\begin{gathered}
A(\mathbf{k}, s)=\frac{s}{m}\left(\cos k_{1}+\ldots+\cos k_{m}\right), \\
S(W(\mathbf{q}))=\frac{1}{N^{m-1} L} \sum_{\substack{q_{2}, \ldots, q_{m} \\
|n|<L / 2}} W\left(q_{1}-\frac{2 \pi n}{L}, q_{2}, \ldots, q_{m}\right) .
\end{gathered}
$$

Let us select uniform starting conditions, i.e., $W_{0}(\mathbf{k})=\delta\left(k_{1}\right) \ldots \delta\left(k_{m}\right)$ and proceed to limit $N \rightarrow \infty$ :

$$
W(\mathbf{k}, s)=\frac{\delta(\mathbf{k})}{1-A(\mathbf{k}, s)}-\frac{A(\mathbf{k}, s)}{1-A(\mathbf{k}, s)} \frac{1}{(1-s)} \times\left(\sum_{q_{1}} \int \ldots \int \frac{d q_{2} \ldots d q_{m}}{1-\frac{s}{m}\left(\cos q_{1}+\ldots+\cos q_{m}\right)}\right)^{-1}
$$


where $q_{1}$ takes on the values $q_{1}=k_{1}-\frac{2 \pi n}{L},|n|<L / 2$. The second term, which enters into the expression with the minus sign, describes particle decay at traps. We are interested in the zeroth harmonic of this term, which determines the probability of the reaction

$$
W(s)=\frac{s}{(1-s)^{2}} \times\left(\sum_{q_{1}} \int \ldots \int \frac{d q_{2} \ldots d q_{m}}{1-\frac{s}{m}\left(\cos q_{1}+\ldots+\cos q_{m}\right)}\right)^{-1} .
$$

For further consideration of the problem, we must evaluate the integral

$$
I(\alpha)=\int \cdots \int \frac{d q_{2} \ldots d q_{m}}{\alpha-\left(\cos q_{2}+\ldots+\cos q_{m}\right)},
$$

where $\alpha=\frac{m}{s}-\cos q_{1}$. This function can have poles only on two points $\alpha= \pm(m-1)$, i.e. $s_{1,2}=\frac{m}{\cos q_{1} \pm(m-1)}, \quad s_{1,2} \geq 1$. Integral (13) in these regions is determined by the values of $q_{i}$, which are nearly zero; therefore, $\cos ()$ in the integrand can be expanded. The change $\cos q_{i} \approx 1-\frac{1}{2} q_{i}^{2}$ corresponds to the changing from the lattice to the continuous limit with respect to the $i$ th coordinate. In the vicinity of the point $\alpha=m-1, \epsilon=\alpha-m+1$ we have

$$
I_{m}(\epsilon)=2 \int \ldots \int \frac{d q_{2} \ldots d q_{m}}{2 \epsilon+q_{2}^{2}+\ldots+q_{m}^{2}} .
$$

Changing to spherical coordinates $\left(S_{m 1}\right.$ is the area of the sphere in the $(m 1)$-dimensional space, $A$ is some constant, $A \sim$ ), we obtain

$$
I_{m}(\epsilon) \approx 2 S_{m-1} \int_{0}^{A} \frac{q^{m-2} d q}{2 \epsilon+q^{2}} .
$$

The values of $I_{m}(\epsilon)$ at different values of $m$ are

$$
I_{m}(\epsilon)=\left\{\begin{array}{cc}
\frac{2 \pi^{2}}{\sqrt{2 \epsilon}}, & m=2 ; \\
8 \pi \log \left(1+\frac{A^{2}}{2 \epsilon}\right), & m=3 ; \\
\sim A^{m-3}, & m \geq 4 .
\end{array}\right.
$$

The probability of decay at traps within these designations is

$$
W(s)=\frac{m}{L(1-s)^{2} \frac{1}{L} \sum_{p=-L / 2}^{L / 2} I\left(\frac{m}{s}-\cos \frac{2 \pi p}{L}\right)} .
$$

The integrand in the function $W(t)$ has singularities $s^{t}$ and $(1-s)^{2}$. We are interested in the behavior of $W(t)$ governed by the vicinity of the point $s=1$, which corresponds to $\alpha=m-\cos q_{1}$. Hence, the expression for the generating function $W(s)$ within a numerical multiplier can be rewritten as follows:

$$
W(s) \sim \frac{1}{(1-s)^{2} \sum_{p=-L / 2}^{L / 2} I_{m}\left(\frac{m}{s}-\cos \frac{2 \pi p}{L}-m+1\right)}
$$


The time dependence $W(t)$ is restored from the generating function $W(s)$ by inverse Laplace transform (8). The contour integral depends on singular points of the integrand, and the pole at the point $s=1$ makes the largest contribution. Recall that the probability $W(t)$ of particle decay unambiguously characterizes the decrease in the reactant concentration with time and, thus, determines the effective rate constant of the chemical reaction. Further calculations will be based on the Tauberian theorem, which readily provides asymptotic estimations in the cases of interest without explicit use of the inverse Laplace transform.

Reactions with $m=1$ and $m \geq 4$. Retaining only the divergent part in expression(12), we obtain the asymptotics

$$
W_{m=1}(t \rightarrow \infty) \sim \sqrt{t}
$$

In the case of $m \geq 4$ the result is also readily obtained because $I_{m} \sim$ const in the region of $s=1$. Therefore, the behavior of the function at large times is governed by the pole $\frac{1}{(1-s)^{2}}$, which corresponds to

$$
W_{m \geq 4}(t \rightarrow \infty) \sim t
$$

Reactions with $\mathbf{m}=\mathbf{2}$. The behavior of $W(t)$ at $m=2$ is more interesting. In this case, the generating function $W(s)$ of the absorption probability is as follows:

$$
W(s) \sim \frac{1}{(1-s)^{2} \sum_{p=-L / 2}^{L / 2}\left(2-s \cos \frac{2 \pi p}{L}-s\right)^{-1 / 2}} .
$$

Applying the Tauberian theorem and replacing the sum with the integral, we obtain

$$
W_{m=2}(t) \sim \frac{t}{\sqrt{t}+\sigma^{-1}+\frac{2 L}{\pi}\left[\log 4-\log \left(\frac{\pi}{L}+\sigma\right)\right]},
$$

where $\sigma=\sqrt{\frac{\pi^{2}}{L^{2}}+\frac{1}{t}}$. In the two limiting cases

$$
W_{m=2}(t) \sim \begin{cases}\frac{t}{t^{1 / 2}+\operatorname{const}} \sim \sqrt{t}, & t \gg \frac{L^{2}}{\pi^{2}} \\ \frac{t}{L \log 8 t}, & 1 \ll t \ll \frac{L^{2}}{\pi^{2}} .\end{cases}
$$

Reactions with $\mathbf{m}=3$. Expression (16) for $I_{m=3}$ gives the correct relationship; however, it contains an undefined constant, which complicates estimations. By the integration of Eq. (13) without expanding cosines in series, we can obtain the more accurate formula

$$
I(\alpha) \approx \pi \log \left(\frac{16}{3-2 s-s \cos q}\right) .
$$

The asymptotics for $t \rightarrow \infty$ can be obtained directly by applying the Tauberian theorem to generating function (17), which leads to the following expression:

$$
W_{m=3}(t) \approx \frac{t}{C+\log t-\log \left(\frac{3}{t}+\frac{2 \pi^{2}}{L^{2}}\right)} .
$$


where $C=5 L \log 2+4 \log \left(\frac{2 \pi}{L}\right)-4-\log \frac{4}{3}$.

Absorption probabilities that were obtained above are insufficient for the exact calculation of the reaction rate but are directly related to the Smoluchowski constant $K_{S m o l}(t)=\frac{d W(t)}{d t}$. Indeed, this constant by defi- nition is the probability of the decay of a solitary particle (i.e., without inclusion of cooperative effects) at the catalyst trap. Within the Smoluchowski approximation, the concentration of particles in the polymolecular reaction of $m$ particles is determined from the kinetic equation

$$
\frac{d C(t)}{d t}=-K_{S m o l}(t) C_{t r} C^{m}(t)
$$

After simple calculations, we obtain $\left(C_{0}=C(t=0), m>1\right)$

$$
C(t)=\left(C_{0}^{1-m}+(m-1) C_{t r} W(t)\right)^{-1 /(m-1)} .
$$

The Smoluchowski approximation is valid in cases when stirring in the system due to the diffusion of particles is a slower process than the chemical reaction event. That is, if the motion of particles is nearly absent, the chemical reaction event involves only the particles that are occasionally the closest to each other. This is the reason why the Smoluchowski approximation is adequate at a low density of particles in the system, when the free diffusion path of particles is sufficiently large compared to the time of the chemical reaction event. To demonstrate the validity of the mean field approximation in the problem under consideration for any distance $L$ between the catalytic centers, it is sufficient to show that the Smoluchowski theory works in the two limiting cases: (a) at $L=1$, i.e., when the reaction occurs each time on the encounter of a pair of particles and, consequently, is independent on the position of the catalytic center; and (b) at $L \rightarrow \infty$, i.e., when the system contains a solitary catalytic center. The exact solution of the many-particle problem in case (a) for bimolecular reactions was reported in [5, 6]. As noted in the introduction, the exact and mean-field solutions have the same asymptotics and differ only by a numerical coefficient. For the case (b), the decrease in concentration $c(t)$ because of the chemical reaction can be easily estimated $(m \geq 2)$ :

$$
\frac{d c_{\text {chem }}(t)}{d t}=-K_{\text {chem }} c_{\text {chem }}^{m}(t), \quad \Rightarrow \quad c_{\text {chem }}(t) \sim \frac{c_{0}}{K_{\text {chem }}} t^{-1 /(m-1)}
$$

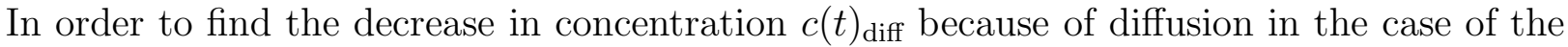
reaction of $m$ particles at a center, we must solve the diffusion equation in $m$-dimensional space, where each coordinate corresponds to the concentration of one of the particles involved in the reaction. As can be easily seen,

$$
\begin{array}{ll}
c_{\text {diff }}(t) \sim c_{0} \frac{\log t}{t} & (m=2) \\
c_{\text {chem }}(t) \sim c_{0} \text { const } & (m=3,4, \ldots)
\end{array}
$$

For all $m \geq 2$, the following condition is fulfilled: after some instant of time $t=t\left(c_{0}, K_{\text {chem }}\right)$, the concentration because of diffusion is larger than the concentration because of the chemical reaction, and, consequently, the rate of diffusion is lower than the rate of the chemical reaction, which means that the Smoluchowski approximation is valid. 
Of particular interest are the relationships $W_{m}(t)$ at large times $(t \rightarrow \infty)$, namely, expressions (20), (23) and (25). As can be seen from the corresponding formulas, the probability of reaction $W_{m}(t)$ is independent of the period of the lattice of catalytic centers. This means that in the given asymptotic mode the probability of the reaction on the lattice with the catalyst placed in each lattice site is the same as on the lattice with the catalyst placed in sparse sites. Recall that this result was first formulated in [1] within the random phase approximation. Thus, our work can be considered as rigorous proof of the effect, which has promising technical applications. We are grateful to G. Oshanin for helpful discussions of the work. The work was supported by the Russian Foundation for Basic Research, project No. 00-15-99302.

\section{References}

[1] G. Oshanin and A. Blumen, J. Chem. Phys. 108 (1998), 1140

[2] M. V. Smoluchowski, Z. Phys. Chem. B 35 (1924), 113

[3] G. Oshanin, M. Moreau, and S. Burlatsky, Adv. Colloid Interface Sci. 49 (1994), 1

[4] S. F. Burlatsky and A. A. Ovchinnikov, Zh. Eksp. Teor. Fiz. 92 (1987), 1618 [Sov. Phys. JETP 65 (1987), 908]

[5] D. C. Torney and H. M. McConnel, J. Phys. Chem. 87 (1983), 1941

[6] A. A. Lushnikov, Phys. Lett. A 120 (1987), 135

[7] B. Ya. Balagurov and V. G. Vaks, Zh. Eksp. Teor. Fiz. 65 (1973), 1939 [Sov. Phys. JETP 38 (1973), 968]

[8] T. C. Lubensky, Phys. Rev. A 30 (1984), 2657

[9] S. R. Renn, Nucl. Phys. B 275 (1986), 273

[10] C. Monthus, G. Oshanin, A. Comtet, and S. F. Burlatsky, Phys. Rev. E 54 (1996), 231

[11] G. C. Bond, Heterogeneous Catalysis: Principles and Applications (Clarendon, Oxford, 1987).

[12] D. Avnir, R. Gutfraind, and D. Farin, in Fractals in Science, Ed. by A. Bunde and S. Havlin (Springer-Verlag, Berlin, 1994). 